\title{
A 40 GHZ POLARIZATION MAINTAINING PICOSECOND MODELOCKED FIBER LASER EMPLOYING PHOTONIC CRYSTAL FIBER
}

\author{
Kazi S. Abedin ${ }^{1}$ and Fumito Kubota ${ }^{2}$ \\ ${ }^{I}$ National Institute of information and Communications Technology, 4-2-1, Nukui-Kita, \\ Koganei-Shi,Tokyo 184-8795JAPAN, abedin@nict.go.jp \\ ${ }^{2}$ National Institute of information and Communications Technology, 4-2-1, Nukui-Kita, \\ Koganei-Shi,Tokyo 184-8795 JAPAN, kubota@nict.go.jp
}

\begin{abstract}
We demonstrate a harmonically mode-locked dispersion-managed polarization-maintaining erbium fiber laser that uses photonic crystal fiber for nonlinear pulse compression. The high nonlinearity and large anomalous dispersion of the PCF resulted in significant reduction in the cavity length and increased the long-term stability. The laser cavity, only $36-\mathrm{m}$-long, yielded stable $1.3 \mathrm{ps}$ pulses at a $40-\mathrm{GHz}$ repetition-rate, with supermode noise suppression of over $60 \mathrm{~dB}$.
\end{abstract}

\section{INTRODUCTION}

Compact tunable sources providing picosecond pulses with tens of $\mathrm{GHz}$ repetition rates and low timing jitter have high demands in many application such as, high speed optical communication systems, optical analog-to-digital conversion and so on. Harmonically active modelocking of fiber lasers is a very useful technique for generating picosecond pulses from fiber lasers with repetition rates of tens to even over a hundred $\mathrm{GHz}$ [1-7]. Mode-locked fiber lasers using the erbium-doped fibers (EDF) as the gain medium have been studied most extensively as they produce pulses in the $1.55 \mu \mathrm{m}$ communication window where the silica fibers exhibit the lowest loss. Technically, short pulse generation at GHzrepetition-rate in erbium doped fiber laser is conveniently realized by taking 
advantage of the high saturation power and the availability of optical fibers with both normal and anomalous dispersion characteristic. One can appropriately manage the cavity-dispersion to favor intracavity nonlinear processes, such as the formation of optical solitons. Operation of the laser in the soliton regime not only yields pulses shorter than the Kuizenga-Siegman limit [8], but also ensures pulsedropout which is particularly important for application in error-free optical communication.

In the high-repetition-rate (10 GHz or above), picosecond pulses can be generated by soliton-effect compression in a long segment of suitable anomalous dispersion fiber placed inside the cavity. Using dispersion shifted fiber (DSF) of $190 \mathrm{~m}$ long inside a laser cavity, solitons with widths of $2.7 \mathrm{ps}$ at $10 \mathrm{GHz}$-rate [2] and 0.9 ps at $40-\mathrm{GHz}$ rate were generated [6]. Recently, highly nonlinear DSF fibers were also deployed with an aim to reduce the length of nonlinear fiber, and indeed 1.2 ps pulses at $40 \mathrm{GHz}$-rate were obtained from an actively mode-locked fiber laser that used $100 \mathrm{~m}$ of highly nonlinear DSF fiber [4].

Alternatively, picosecond pulses can also be produced from a dispersion managed (DM) laser cavity, which has fiber segments with large local dispersion, but a small anomalous path-averaged dispersion. In a DM laser a pulse spends much of its time in a stretched state and experiences a lower effective nonlinearity than a fundamental soliton pulse in a comparable uniform dispersion cavity [9]. This allows pulses to circulate with higher energy in the cavity, yielding higher output power. Pulses with a width of $1.3 \mathrm{ps}$, timing jitters of only $10 \mathrm{fs}$, and pulse dropout ratio smaller than $10^{-14}$ were generated at $10 \mathrm{GHz}$ rate from a $\mathrm{DM}$ sigma fiber laser $[3,10]$.

We have recently demonstrated a mode-locked erbium fiber laser which uses a polarization maintaining PCF (PM-PCF) with high nonlinearity and anomalous dispersion for nonlinear pulse compression [11]. The high nonlinearity and large anomalous dispersion of the PCF made nonlinear pulse compression achievable in fiber only $10-\mathrm{m}$ long. Highly-stable pulses with $1-\mathrm{ps}$-width and $10-\mathrm{GHz}$ repetitionrate were obtained over a range of $1535-1560 \mathrm{~nm}$. In this paper, we demonstrate successful operation of PCF-based compact fiber laser at 40-GHz-repetition-rate. We have produced $\sim 1.3$ ps pulses at $40-\mathrm{GHz}$ repetition rate with supermode noise suppression better than $60 \mathrm{~dB}$ and output power of over $14 \mathrm{mw}$.

\section{EXPERIMENTAL SETUP}

The experimental setup of the mode-locked fiber ring laser is shown in Fig. 1. The cavity consisted of a 20-m long Er-doped PANDA fiber, a Mach-Zehnder modulator, an optical isolator, a tunable bandpass filter (3-dB-bandwidth: $8 \mathrm{~nm}$ ), a $30 \%$ output coupler, and a 10-m-long polarization maintaining PCF (PM-PCF) 
section. The Er-fiber had an un-pumped absorption coefficient of $3.28 \mathrm{~dB} / \mathrm{m}$ and dispersion of $-54 \pm 5 \mathrm{ps} / \mathrm{nm} / \mathrm{km}$ at $1550 \mathrm{~nm}$. The PM-PCF, manufactured by Mitsubishi Cable Industries, had a Ge-doped core that was surrounded by a hexagonal array of holes. The cross section of the PM-PCF is shown in the inset of Fig 1 . The fiber had a zero-dispersion wavelength at $876 \mathrm{~nm}$, and exhibited a dispersion parameter of 104 and $126 \mathrm{ps} / \mathrm{nm} / \mathrm{km}$, for the slow and fast axes respectively. The PM-PCF had a nonlinear coefficient of $39.5 \mathrm{~W}^{-1} \mathrm{~km}^{-1}$ and a loss coefficient of $16 \mathrm{~dB} / \mathrm{km}$ (at $1550 \mathrm{~nm}$ ). To facilitate optical coupling, both ends of the PM-PCF were fusion-spliced to standard PANDA fiber through a mode-field converter, which yielded a loss/splice of only $1.6 \mathrm{~dB}$. The cavity also employed a fused fiber polarization beam splitter (PBS) that restricted oscillation to slow axes in the cavity. The doped fiber and PM-PCF within the laser cavity mapped a dispersion-managed (DM) periodic system with large dispersion variation. The dispersion map of the cavity, which mostly consisted of the erbium fiber and the PCF is shown in Fig. 2. The average value of dispersion in the 36-m-long cavity was $1.4 \mathrm{ps} / \mathrm{nm} / \mathrm{km}$, and the fundamental cavity repetition rate was $5.6 \mathrm{MHz}$.

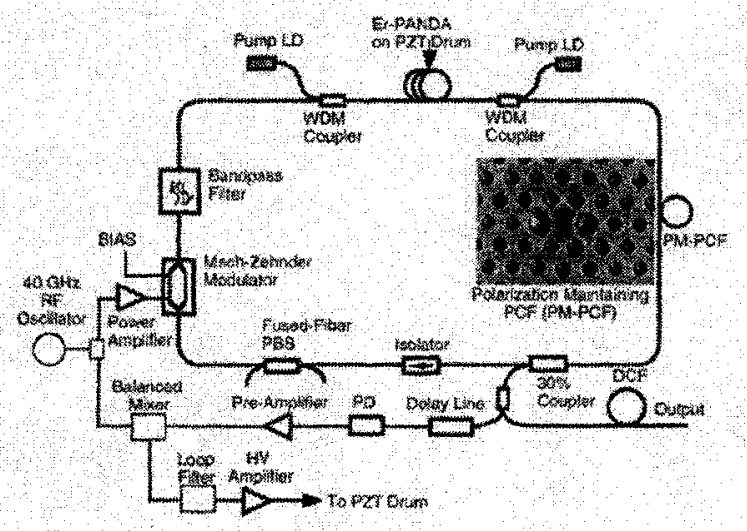

Figure 1. Experimental setup

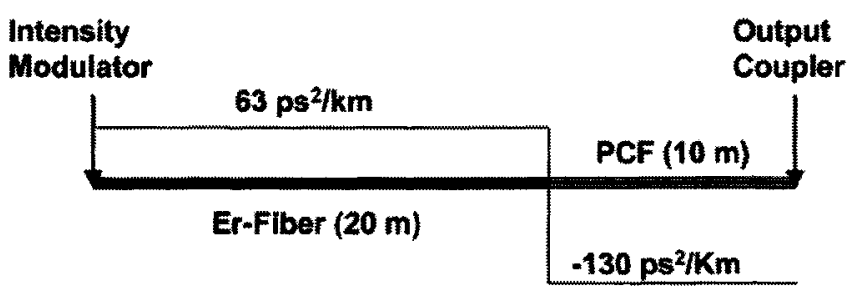

Figure 2. Dispersion map of the laser. 
For modelocking at a 40-GHz-repetition-rate, we directly drove the modulator using an RF oscillator and a narrow band RF power amplifier. We also stabilized the cavity by controlling the length of the Er-PANDA fiber that was wound around a cylindrical piezoelectric transducer (PZT). The error signal necessary for active feedback was obtained from the phase of the output pulses relative to the clock signal available at the IF output port of the double balanced mixer. The error signal was processed with proportional and integration control circuitry, amplified and applied to the PZT [12].

\section{RESULTS}

Modelocking of the laser was achieved at a repetition rate of $\sim 40 \mathrm{GHz}$ by carefully adjusting the oscillator frequency to match a harmonic of the fundamental cavity-repetition-rate and the bias voltage of the modulator. The optical spectrum of the mode-locked pulses is shown in Fig. 3(a), which shows the optical comb of frequencies separated by a frequency equal to pulse repetition rate. The ASE noisefloor was over $10^{-4}$ times lower than the peak of spectrum. The envelope of the optical spectrum could be fitted by a Gaussian-like envelope with an FWHM bandwidth of $2.69 \mathrm{~nm}$. In soliton systems that has largely differing dispersions [3, $10]$, optical spectrum with the Gaussian rather than sech-profile are commonly seen. In our experiment, we obtained output pulses with nonzero chirp that was accounted for by external chirp-compensation using dispersion compensating fiber (DCF) of about a 4-m-long. The autocorrelation trace of the shortest pulse thus obtained is shown in Fig. 3(b), which yielded an FWHM pulsewidth of 1.29 ps.
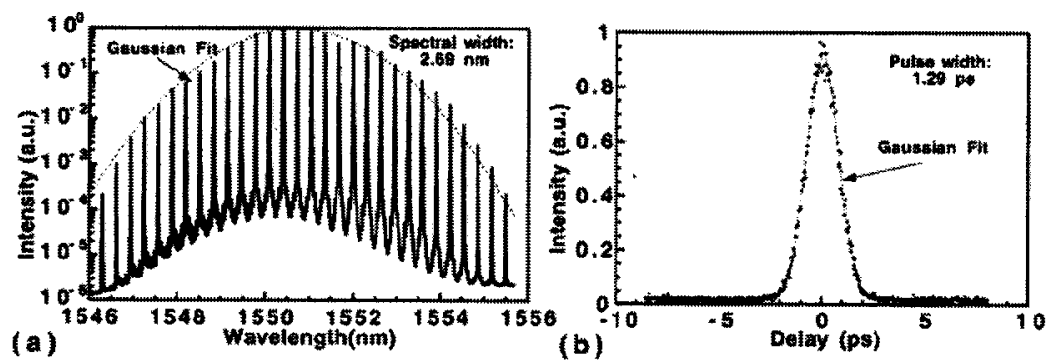

Figure 3. Output pulses. a) Optical spectrum. b) Autocorrelation trace.

Figure 4(a) represents the autocorrelation trace measured over a longer time scale, which shows periodic pulse trains separated by about $\sim 25 \mathrm{ps}$, in consistent with the RF modulation frequency. The RF spectrum of the output pulses showed a frequency component equal to pulse repetition-rate of $39.463 \mathrm{GHz}$. The supermode 
noise was suppressed by more than $60 \mathrm{~dB}$. The average output power was 14.4 $\mathrm{mW}$, which was limited by the power of the pump LDs. The wavelength of the output could be changed through the use of the tunable bandpass filter.
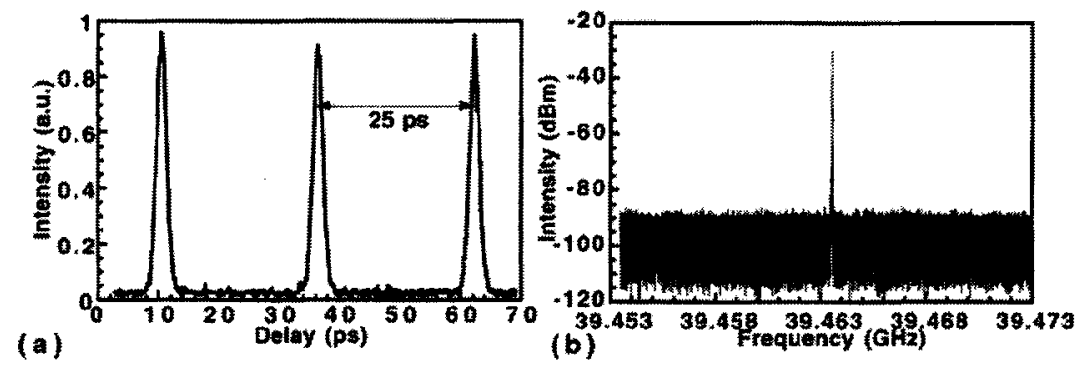

Figure 4. Output pulses. a) Auto/cross correlation trace, b) RF spectrum.

It is known that in a fiber system with dispersion maps that have large deviations of the local dispersion from the average-dispersion, the pulses have enhanced energy relative to solitons in a system with uniform dispersion that is equal to the path-averaged dispersion of the map [13]. The dispersion map strength factor $\gamma_{S}=2 \quad\left|\left(\beta_{2 n}-\beta_{2 a v g}\right)_{n}\right| / \tau_{F W H M}{ }^{2}$ of the DM soliton laser, where $\beta_{2 \mathrm{n}}$ and $l_{\mathrm{n}}$ is the group velocity dispersion and length of the $n$th fiber segment forming the cavity, $\tau_{\mathrm{FWHM}}$ is the shortest FWHM pulse duration, $\boldsymbol{\beta}_{2 \text { avg }}$ is the average dispersion, can be determined using a value of $1.29 \mathrm{ps}$ for $\tau_{\mathrm{FWHM}}$ and the parameters of the PCF and Er-PANDA. We obtain a value for $\gamma_{S}$ of $\sim 3.0$ for the $36-\mathrm{m}$-long dispersion map, while it was 4.5 for the same laser used for producing 1.07 ps pulse at $10 \mathrm{GHz}$, repetition rate [11]. Higher value of $\gamma$ and, correspondingly shorter pulses with larger output power are expected by using pump LDs with higher power and further optimization of the dispersion map of the cavity.

\section{CONCLUSIONS}

In this paper we have demonstrated the application of highly nonlinear and anomalously dispersive PCF in compact pulse sources that generate tunable picosecond pulse at high repetition rate suitable for optical communication. The use of PCF has helped reduction in the cavity length by an order of magnitude. We successfully produced 1.3 ps pulses at a $40 \mathrm{GHz}$ repletion rate, and the supermode noise was suppressed by more than $60 \mathrm{~dB}$. 


\section{REFERENCES}

[1] J. D. Kafka, T. Baer, and D. W. Hall, "Mode-locked erbium-doped fiber laser with soliton pulse shaping," Opt. Lett., vol. 14, pp. 1269-1271, 1989.

[2] M. Nakazawa, E. Yoshida, and Y. Kimura, "Ultrastable harmonic and regeneratively mode-locked polarization-maintaining erbium fiber ring laser," Electron. Lett., vol. 30, pp. 1603-1605, 1994.

[3] T. F. Carruthers, I.N. Duling III, " $10-\mathrm{GHz}, 1.3$-ps erbium fiber laser employing soliton pulse shortening," Opt. Lett., vol. 21, pp. 1927-1929, 1996.

[4] B. Bakhshi and P. A. Andrekson, " $40 \mathrm{GHz}$ actively modelocked, polarization maintaining erbium fiber ring laser," Electron. Lett., vol. 36, pp. 411-413, 2000.

[5] D. Ellis, R. J. Manning, I. D. Phillips, and D. Nesset, " $1.6 \mathrm{ps}$ pulse generation at 40 $\mathrm{GHz}$ in phase locked ring laser incorporating highly nonlinear fiber for application to $160 \mathrm{Gbit} / \mathrm{s}$ OTDM networks," Electron Lett., vol. 35, pp. 645-646, 1999.

[6] E. Yoshida, N. Shimizu, and M. Nakazawa, "A 40-GHz 0.9-ps regeneratively modelocked fiber laser with a tuning range of $1530-1560 \mathrm{~nm}$," IEEE Photon. Technol. Lett., vol. 11., pp. 1587-1589, 1999.

[7] K. S. Abedin, M. Hyodo and N. Onodera and, "Active stabilization of a higher-order mode-locked fiber laser operating at a pulse repetition-rate of $154 \mathrm{GHz}$," Opt. Lett., vol. 26, pp. 151-153, 2001.

[8] D. J. Kuizenga and A. E. and Siegman, "FM and AM mode locking of the homogeneous laser - Part I: Theory," IEEE J. Quantum Electron., vol. 6, pp. 694-708, 1970.

[9] K. Tamura, E. P. Ippen, H. A. Haus, and L. E. Nelson, "77-fs pulse generation from a stretched-pulse mode-locked all-fiber ring laser," Opt. Lett., vol.18., pp. 1080-1082, 1993.

[10] T. Carruthers, Irl N. Duling, M. Horowitz, and C. R. Menyuk, "Dispersion management in harmonically mode-locked soliton laser," Opt. Lett., vol. 25, pp. 153$155,2000$.

[11] K. S. Abedin and F. Kubota, "A 10-GHz, 1-ps regeneratively modelocked fibre laser incorporating a highly nonlinear and dispersive photonic crystal fibre for intracavity nonlinear pulse compression," Electron. Lett., 40, pp. 58-60, 2004.

[12] X. Shan, D. Cleland, A. Ellis, "Stabilizing Er fiber soliton laser with pulse phase locking," Electron. Lett., vol. 28, pp. 182-184, 1992.

[13] V. S. Grigoryan, T. Yu, E. A. Golovchenkom, C. R. Menyuk and A. N. Pilipetskii, "Dispersion-managed soliton dynamics," Opt.Lett., vol. 22, pp. 1609-1611, 1997. 